\title{
An Efficient Learning approaches on Alzheimer 's disease using ACCF for Image Feature Extraction Technique
}

\author{
D. Deepa ${ }^{1}$, Dr.Anitha Karthi ${ }^{2}$ \\ Research Scholar, Department of Computer Science and Engineering, Bharath Institute of Higher Education and \\ Research, BIHER, BIST, Bharath University, Chennai, India ${ }^{1}$ \\ Professor, Department of Computer Science and Engineering, Bharath Institute of Higher Education and \\ Research, BIHER, BIST, Bharath University, Chennai, India ${ }^{2}$ \\ deepasaram@gmail.com¹, anithakarthi.cse@bharathuniv.ac.in²
}

\begin{abstract}
:
Now a day's making classification for nuero images of Alzheimers disease and related important progress had been proposed by using shallow learning techniques. The importance of shallow learning not yet to be paid more attention for that the endemic challenge of neuroimaging produced by the insufficient data. In this research work, proposed system has Image enhancement techniques with inductive learning approaches. This research works finds that image feature extraction technique such as Auto color correlogram Filter techniques on Alzheimer's images dataset by implementing statistical learning and ensemble learning approached. An Iterative Classifier Optimizer of Ensemble category gives $93 \%$ of accuracy level, 0.93 of precision value, 0.93 of recall value, 0.94 of receiver operating characteristic curve area under value (ROCAUC) value and 0.94 of precision recall curve value, and it takes time consumption as 1.55 seconds to build a model which is produced an optimal results based on their performance compare with other models. This research works recommends that image feature extraction technique such as Auto color correlogram Filter techniques on Alzheimer's images dataset by implementing Iterative Classifier Optimizer classifier of ensemble model.
\end{abstract}

\section{Keywords: Auto Color Correlogram Filter, Bayes, Alzheimer's disease, and Ensemble}

\section{Introduction}

Alzheimer's disease is the mostly affects the people who are crossing 65 years old and is categorized by continue deterioration of cognitive and memory abilities $[1,2]$.

The Image collections and processing of neuroimaging collected from magnetic resonance imaging, functional MRI, positron emission tomography, and also diffusion tensor imaging, conducted by expert persons. An early detection of Alzheimer's disease and its prodromal stage, moderate cognitive impairment, is critical. A valid diagnosis based on brain imaging is required, and a strong diagnostic system assisted by neuroimaging processing can permit for a more useful and reliable approach, and potentially enlarged diagnostic accuracy.

Traditional methods for examining neuroimaging biomarkers for the testing and analysis of neuropsychiatric diseases relied on mass univariate statistics approach, presumptuous that various brain areas function separately. However, given our present understanding of brain function, this assumption is incorrect. $[2,3]$

Machine learning (ML) approaches that take into consideration interregional correlation have recently been a popular and important part of computer-assisted analytical procedures [4] and utilizes for diagnosis process is fully based on automation of neuropsychiatric diseases. $[5,6]$

This paper is structured as section 2 shows the connected works; section 3 displays the materials and techniques; section 4 provides the findings and discussions; and lastly, section 5 shows the conclusion.

\section{Literature Survey}

Several comprehensive evaluations of medical imaging employing machine learning approaches have been published. Due to their incapacity to extract adaptive features, Support Vector Machine based, automated diagnosis models for neuropsychiatric disorders [7-9] prefer to employ hand-crafted features. The Support Vector Machine approach has given not better outcome on raw data and extracting useful features requires of process. $[10,11]$. 
Shallow learning to use input raw data and allow it to recognize highly additional features in training data set automatically [12]. Deep Learning [4,13-17] works at Level 1, and image feature extraction was quite far from automation process. The use of Convolutional Neural Networks is having numerous disciplines, starting with AlexNet's excellent success on the natural picture classification issue [18].

Early successes in medical image processing were gained in 2D pictures such as retinal and chest X-ray images [19], which were later expanded to 3D images like magnetic resonance imaging. Existing Convolution Neural Networks-based magnetic resonance imaging processes are usually categorized on Level 2. During preprocessing, various works[20,21] segment the grey matter area and subsequently use it as a Convolutional Neural Networks input.

Three Dimensional with Convolutional Neural Networks has dropout, batch normalization, as well residual module regularization techniques. [22-27]. Multimodal DL techniques [4,16,17, and 26,27] have sought to enhance the classification accuracy of AD by using multiple inputs and DL models.

\section{Materials and Methods}

In this segment concentrations on the Materials and methods on this research work. Alzheimer's images lended from Kaggle repository. The below table shows that the description of the collected dataset.

\begin{tabular}{|c|l|c|c|c|c|}
\hline S.No & \multicolumn{1}{|c|}{ Category } & $\begin{array}{c}\text { Actual } \\
\text { Image } \\
\text { Size }\end{array}$ & $\begin{array}{c}\text { Processed } \\
\text { Image Size }\end{array}$ & $\begin{array}{c}\text { Number of } \\
\text { Images }\end{array}$ & $\begin{array}{c}\text { Sample Size } \\
\text { (Random with balanced } \\
\text { data) }\end{array}$ \\
\hline 1 & Non Demented & $176 \times 208$ & $256 \times 256$ & 1792 & 50 \\
\hline 2 & Very Mild Demented & $176 \times 208$ & $256 \times 256$ & 2560 & 16 \\
\hline 3 & Mild Demented & $176 \times 208$ & $256 \times 256$ & 717 & 17 \\
\hline 4 & Moderate Demented & $176 \times 208$ & $256 \times 256$ & 52 & 17 \\
\hline \multicolumn{2}{r|}{ Total } & & & $\mathbf{1 0 0}$ \\
\hline
\end{tabular}

Table 1: Meta data of Dataset

\section{Methods:}

The succeeding methods are applied in this research work.

1) Borrowed dataset

2) Data preprocessing

3) Apply Auto Color Correlogram Filter

4) Apply for Bayes and Meta machine learning algorithms:

a)Bayes : Bayes Net(BN), Naïve Bayes Multinomial (NBM) and Naïve Bayes Multinomial Updateable(NBMU)

b)Meta:AdaBoostM1,Bagging and Iterative Classifier Optimizer(ICO)

5) To get Optimal results

6) Find a best Model

To produce an efficient outcome, these strategies were applied in one of the top and open source programmes, Weka 3.9.5. This study uses only $10 \%$ of the whole dataset and uses tenfold cross validation for all categories. 


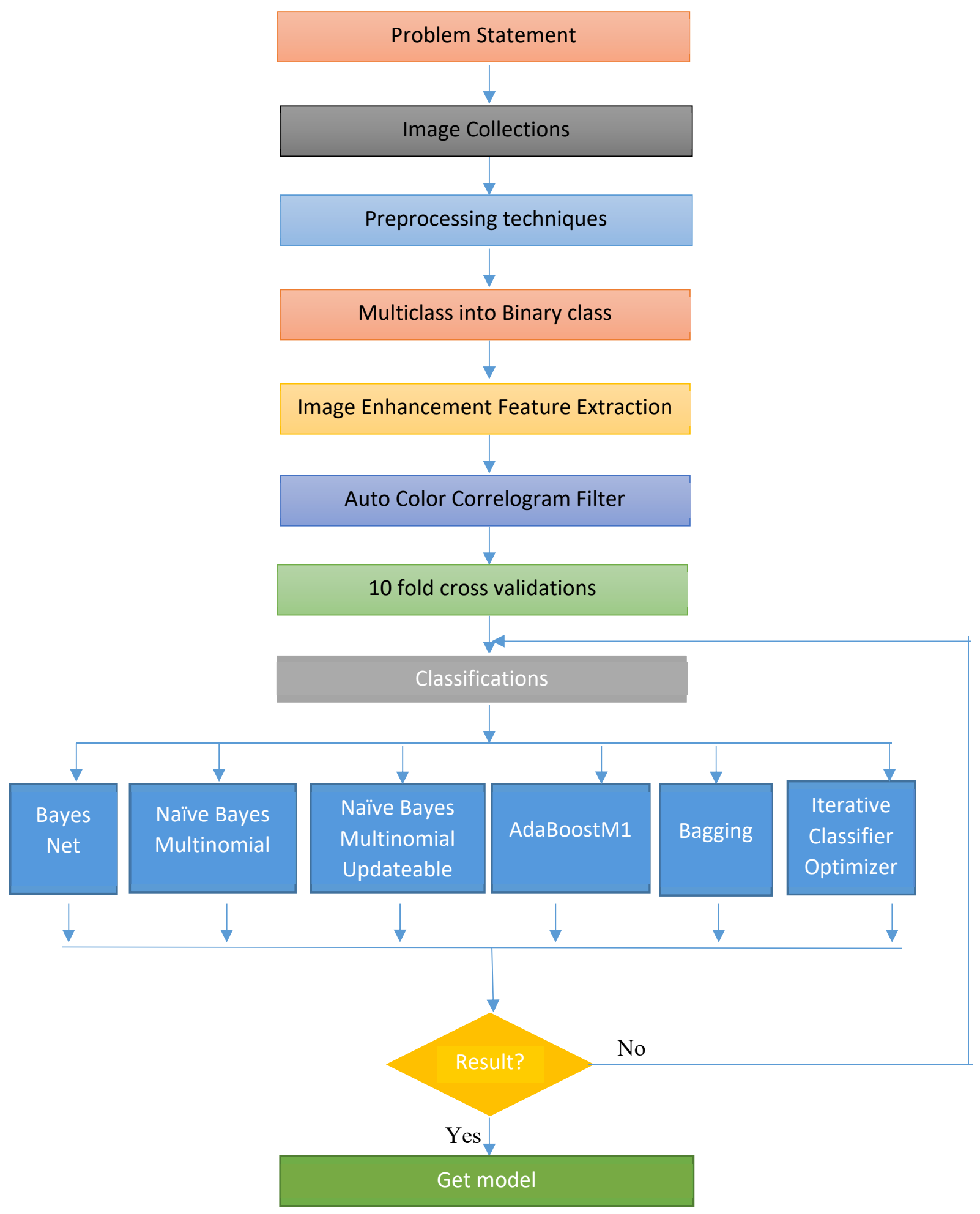

Figure 1: Proposed System 


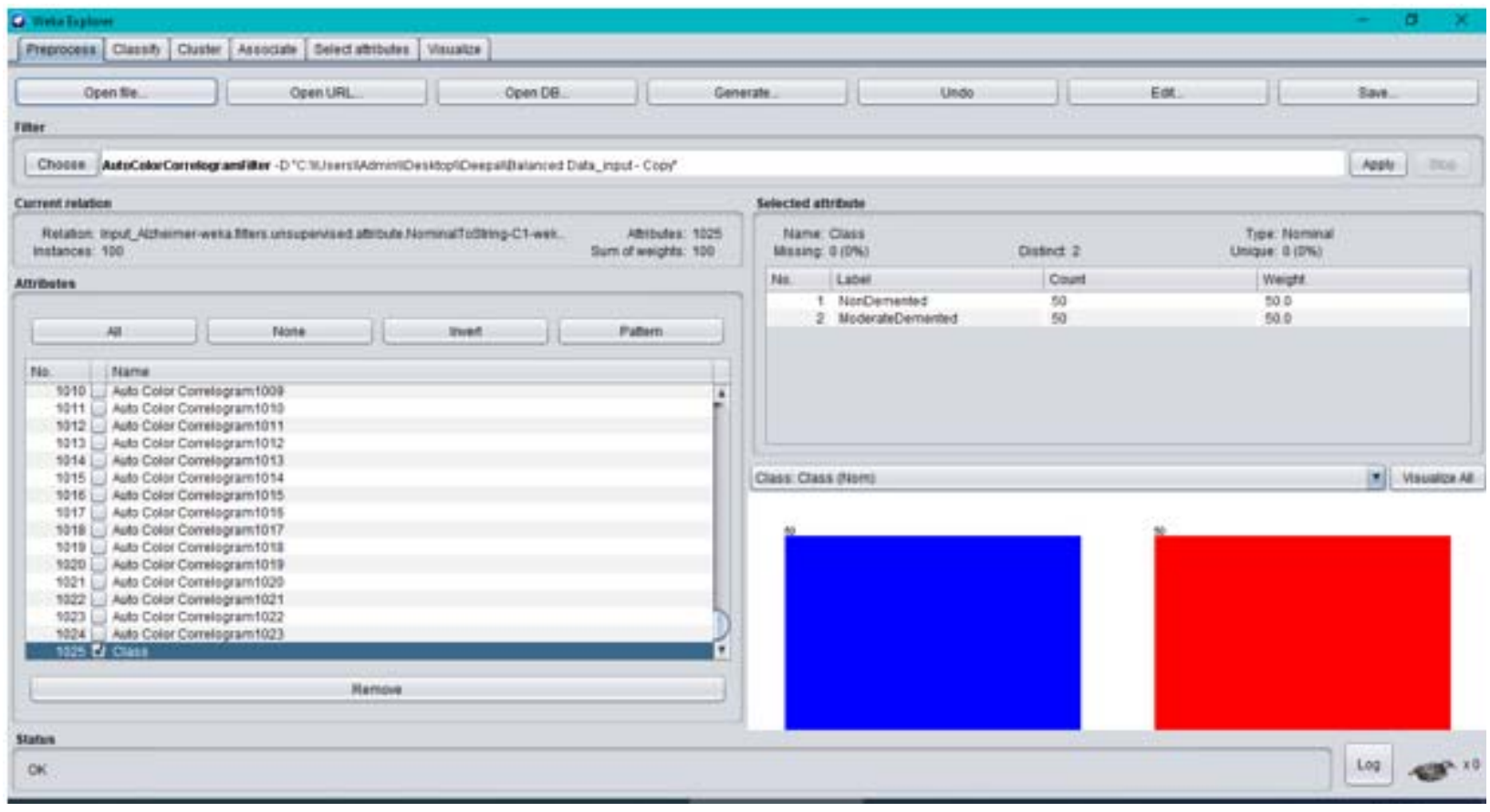

Figure 2: Class distribution in Weka

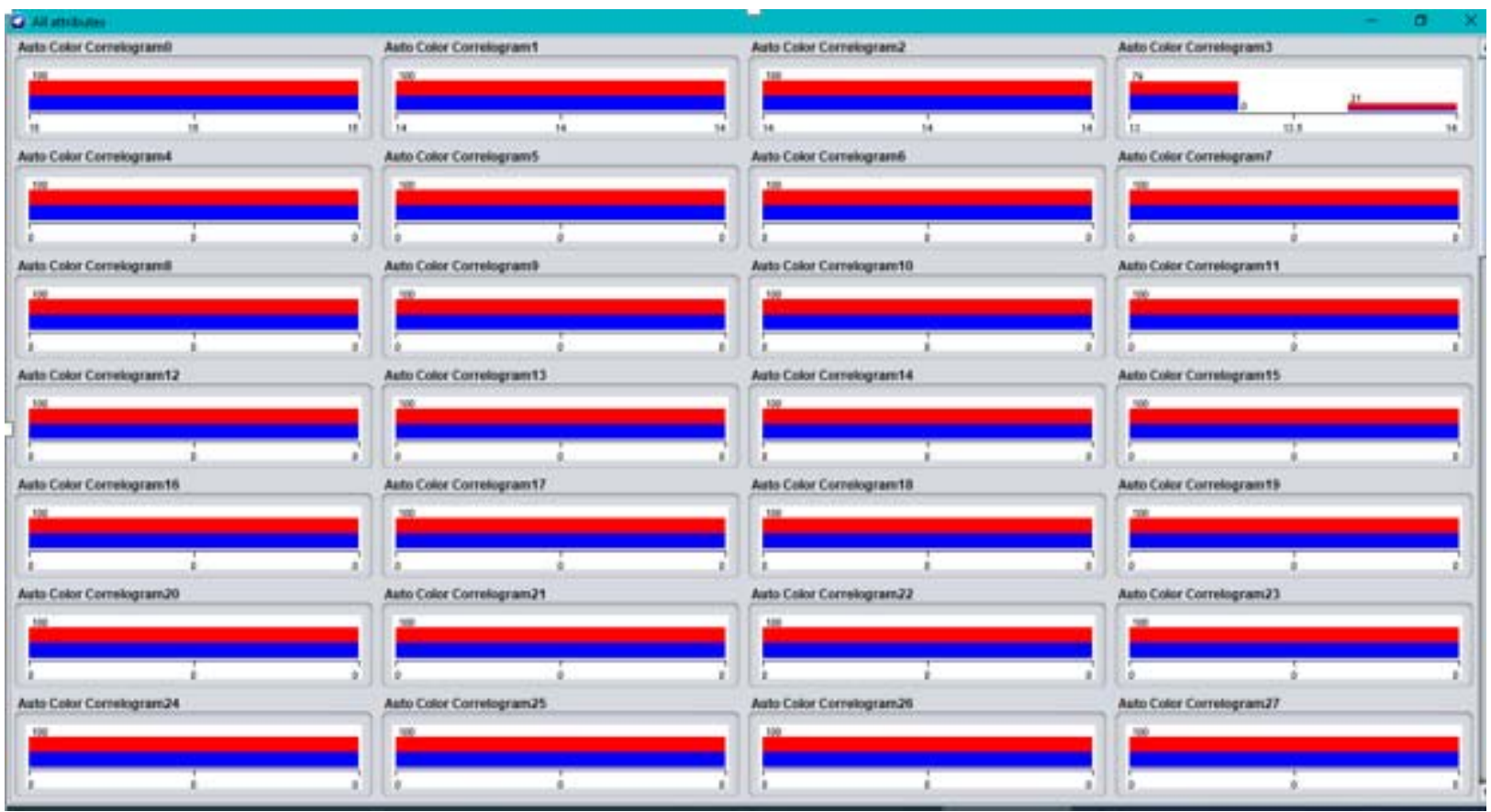

Figure 3: Image enhancement technique (Auto Color Correlogram Filter) implementation in Alzheimer images 


\begin{tabular}{|c|c|c|c|c|c|c|c|c|}
\hline $\begin{array}{c}\text { S.N } \\
\text { o }\end{array}$ & $\begin{array}{c}\text { Base } \\
\text { Category }\end{array}$ & Classifier & $\begin{array}{c}\text { Time } \\
\text { Taken } \\
\text { to } \\
\text { build } \\
\text { model }\end{array}$ & Accuracy & Precision & Recall & ROC & PRC \\
\hline 1 & Bayes & Bayes Net & 0.16 & $91 \%$ & 0.92 & 0.91 & 0.97 & 0.97 \\
\hline 2 & Bayes & $\begin{array}{l}\text { Naïve Bayes } \\
\text { Multinomial }\end{array}$ & 0.02 & $88 \%$ & 0.89 & 0.88 & 0.94 & 0.94 \\
\hline 3 & Bayes & $\begin{array}{l}\text { Naïve Bayes } \\
\text { Multinomial } \\
\text { Updateable }\end{array}$ & 0.05 & $88 \%$ & 0.89 & 0.88 & 0.94 & 0.94 \\
\hline 4 & Meta & $\begin{array}{c}\text { AdaBoostM } \\
1\end{array}$ & 0.45 & $88 \%$ & 0.88 & 0.88 & 0.94 & 0.93 \\
\hline 5 & Meta & Bagging & 0.61 & $91 \%$ & 0.91 & 0.91 & 0.96 & 0.95 \\
\hline 6 & Meta & $\begin{array}{c}\text { Iterative } \\
\text { Classifier } \\
\text { Optimizer }\end{array}$ & 1.55 & $93 \%$ & 0.93 & 0.93 & 0.94 & 0.94 \\
\hline
\end{tabular}

Table 2: Performance of Bayes and Meta Classifiers

The Bayes Net classifier of the Bayes category has 91\% accuracy, the Nave Bayes Multinomial classifier of the Bayes category has $88 \%$ accuracy, the Nave Bayes Multinomial Updateable Classifier of the Bayes Category has $88 \%$ percent accuracy, the AdaBoostM1 classifier of the Ensemble category has $88 \%$ accuracy, the Bagging classifier of the Ensemble category has 91\% accuracy and Iterative Classifiers Optimizer of meta category has $93 \%$ of accuracy performance.

The Bayes Net classifier in the Bayes category has a Precision value of 0.92, the Nave Bayes Multinomial classifier in the Bayes category has a Precision value of 0.89, the Nave Bayes Multinomial Updateable Classifier has a Precision value of 0.89, the AdaBoostM1 classifier in the Ensemble category has a Precision value of 0.88 , the Bagging classifier in the Ensemble category has a Precision value of 0.91, and the Iterative Classifiers Optimizer of meta category has 0.93 of precision performance.

The Bayes Net classifier in the Bayes category has a recall value of 0.91, the Nave Bayes Multinomial classifier in the Bayes category has a recall value of 0.88 , the Nave Bayes Multinomial Updateable Classifier in the Bayes category has a recall value of 0.88 , the AdaBoostM1 classifier in the Ensemble category has a recall value of 0.88 , the Bagging classifier in the Ensemble category has a recall value is 0.91 of recall performance level and the and Iterative Classifiers Optimizer of meta category has 0.93 of recall performance.

The Bayes Net classifier in the Bayes category has 0.97 of ROC performance value, the Nave Bayes Multinomial classifier in the Bayes category has 0.94 of ROC performance value, the Nave Bayes Multinomial Updateable Classifier in the Bayes category has value of 0.94 of ROC performance value, the Ensemble category's AdaBoostM1 classifier has 0.94 of ROC performance value, the Bagging classifier in the Ensemble category has 0.96 of ROC performance value and the and Iterative Classifiers Optimizer of meta category has 0.94 of ROC performance value.

The Bayes Net classifier in the Bayes category has a precision recall curve value of 0.97, the Nave Bayes Multinomial classifier in the Bayes category has a precision recall curve value of 0.94, the Nave Bayes Multinomial Updateable Classifier has a precision recall curve value of 0.94, the AdaBoostM1 classifier in the Ensemble category has a precision recall curve value of 0.93, and the Bagging classifier in the Ensemble category has 0.95 of PRC performance level and Iterative Classifiers Optimizer of meta category has 0.94 of PRC performance level,

Building a model with the Bayes Net classifier in the Bayes category takes 0.16 seconds. The Bayes category's Nave Bayes Multinomial classifier builds a model in 0.02 seconds. Building a model with the Nave Bayes Multinomial Updateable Classifier takes 0.05 seconds. The Ensemble category's AdaBoostM1 classifier takes 0.45 seconds to construct a model. The Ensemble category's Bagging classifier takes 0.61 seconds to create 
a model, whereas the Ensemble category's Iterative Classifier Optimizer classifier takes 1.55 seconds for building a model.

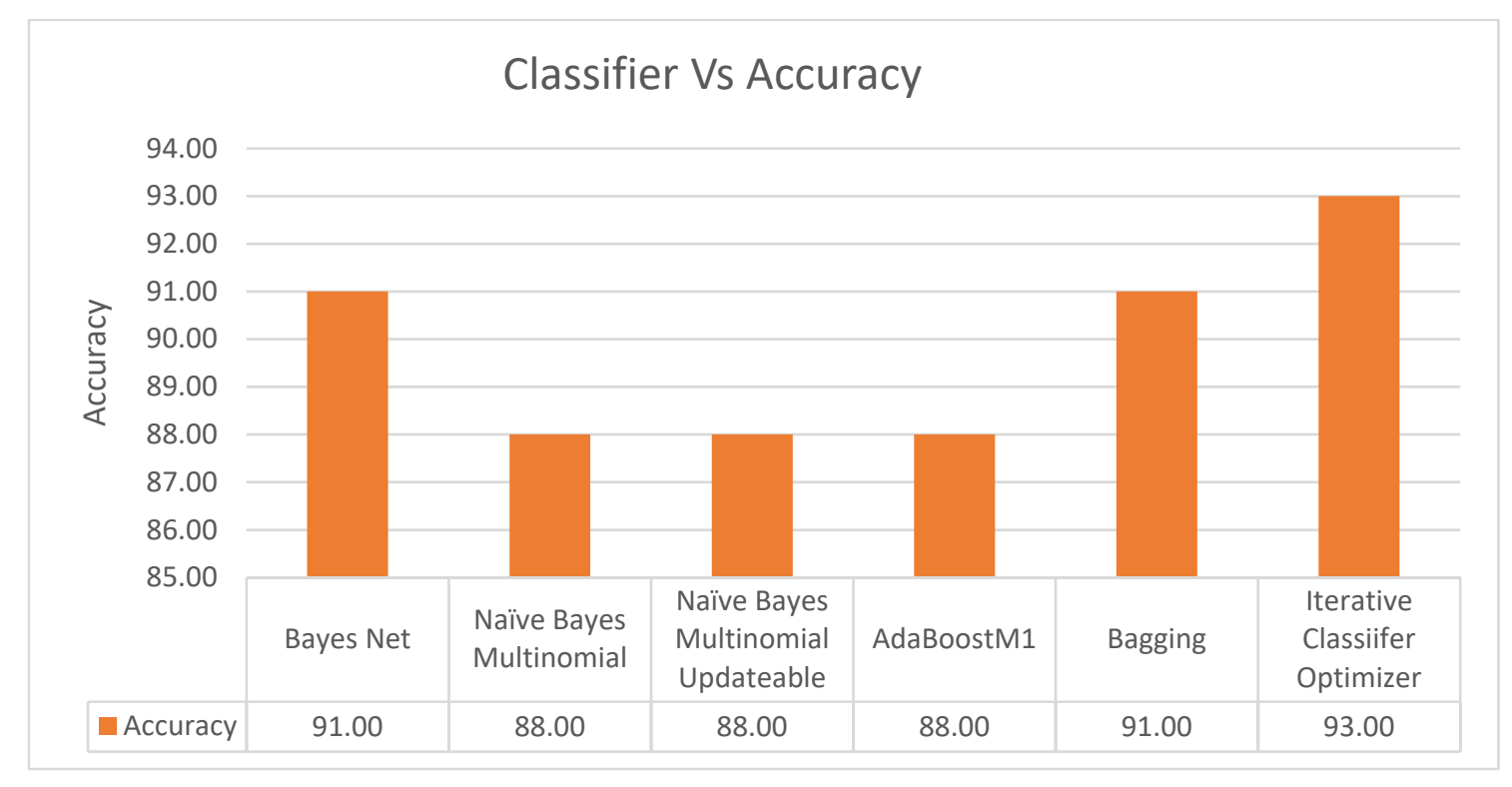

Figure 4: Performance of various classifiers with their accuracies

The above figure 4 shows that the Naïve Bayes Multinomial classifier and Naïve Bayes Multinomial Updateable classifier of Bayes category and AdaBoostM1 classifier of Meta category has same as well least accuracy level which is $88 \%$ of accuracy value. The Bayes Net and Baggig classifier has same which is $91 \%$ of accuracy level. The Iterative Classifier Optimization is having highest accuracy level which is $93 \%$ of accuracy.

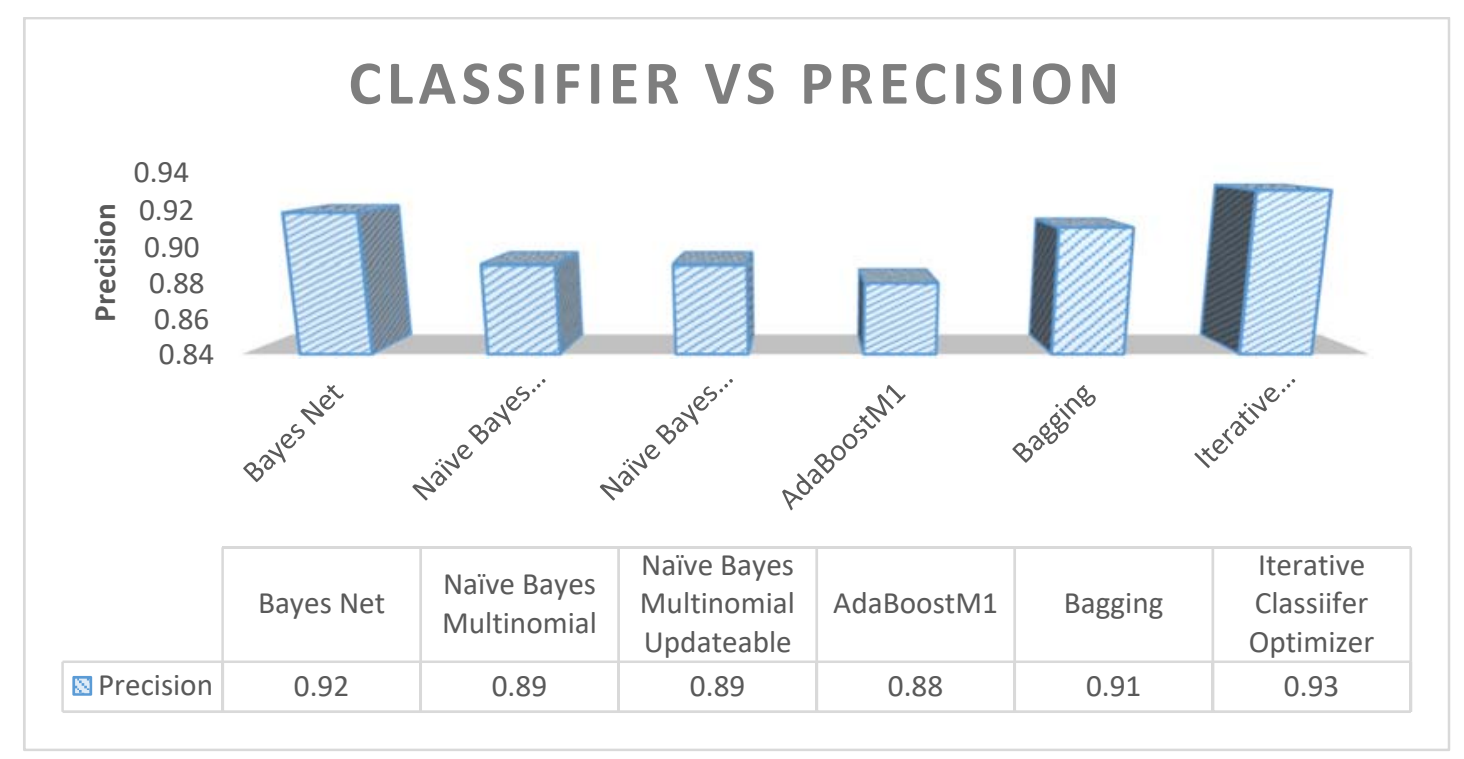

Figure 5: Performance of various classifiers with their Precision values

The above figure 5 shows that the Naïve Bayes Multinomial classifier and Naïve Bayes Multinomial Updateable classifier of Bayes category and AdaBoostM1 classifier of Meta category has more or less same precision value as well least precision level which is $0.89,0.89$ and 0.88 of precision values. The Bayes Net is having 0.92 of precision value. The Bagging classifier is 0.91 of precision level. The Iterative Classifier Optimization is having highest precision level which is 0.93 of precision value. 


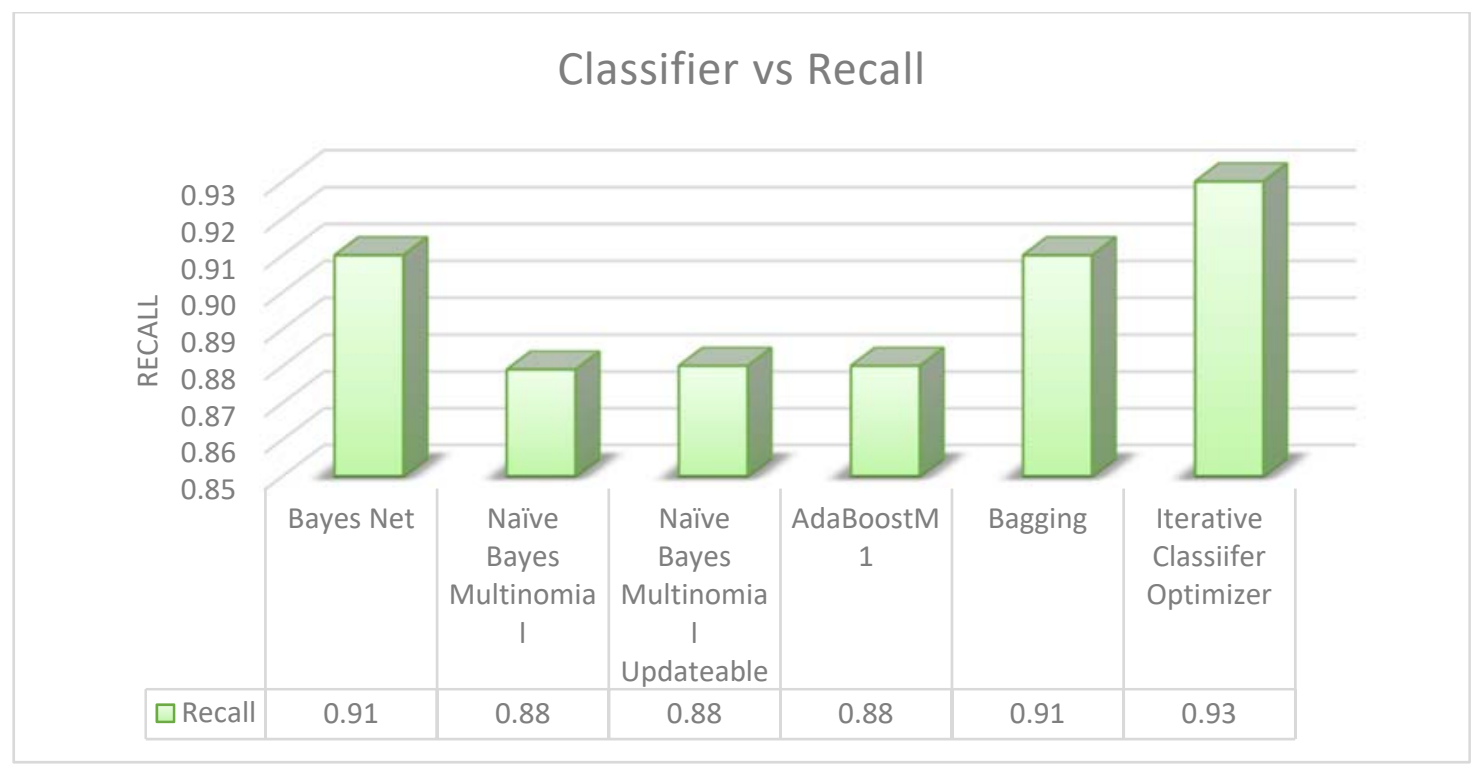

Figure 6: Performance of various classifiers with their Recall values

The above figure 6 shows that the Naïve Bayes Multinomial classifier and Naïve Bayes Multinomial Updateable classifier of Bayes category and AdaBoostM1 classifier of Meta category has same as well least recall level which is 0.88 of recall value. The Bayes Net is having 0.91 of recall value. The Bagging classifier is 0.91 of recall level. The Iterative Classifier Optimization is having highest recall level which is 0.93 of recall value.

\section{CLASSIFIER VS ROC}

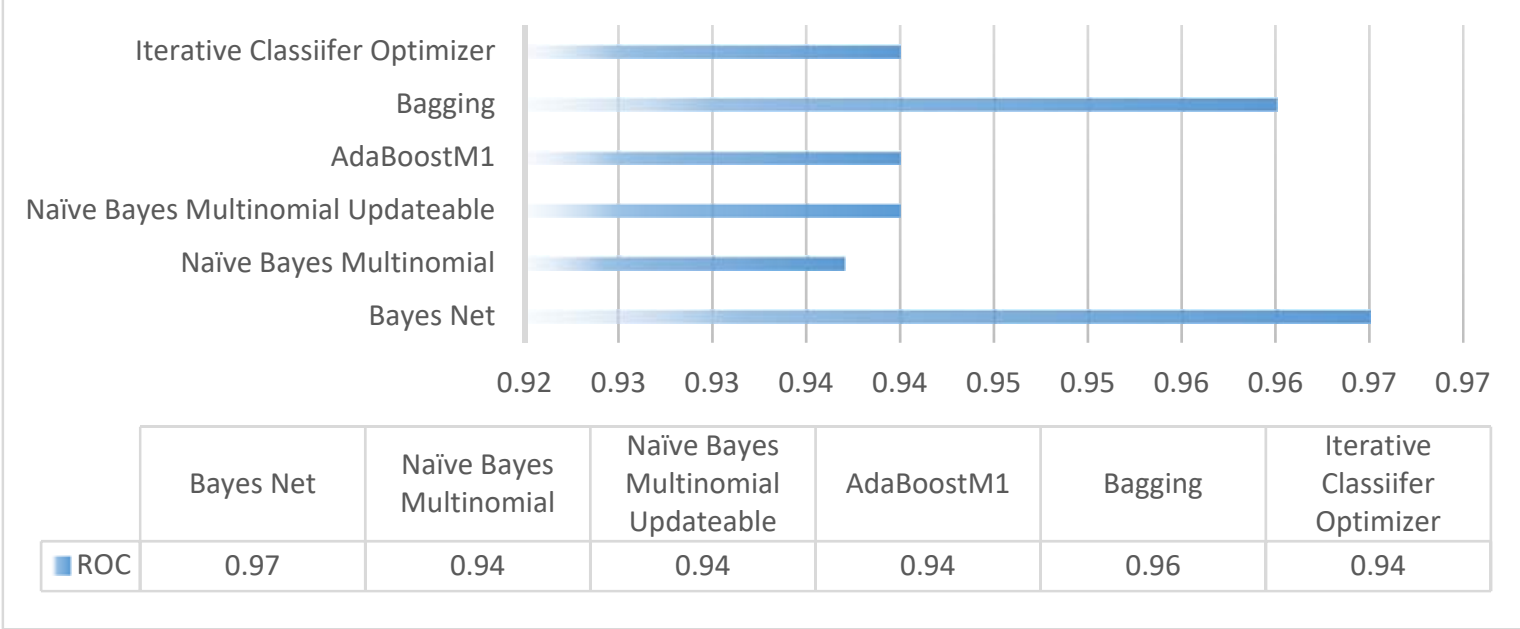

Figure 7: Performance of various classifiers with their ROC values

The above figure 7 shows that the Naïve Bayes Multinomial classifier and Naïve Bayes Multinomial Updateable classifier of Bayes category and AdaBoostM1 classifier of Meta category has same ROC values as well least ROC level which is 0.94 of ROC values. The Bayes Net is having 0.97 of ROC value which is highest ROC value. The Bagging classifier is 0.96 of ROC level. The Iterative Classifier Optimization is having 0.94 of ROC value. 


\section{CLASSIFIER VS PRECISION RECALL CURVE}

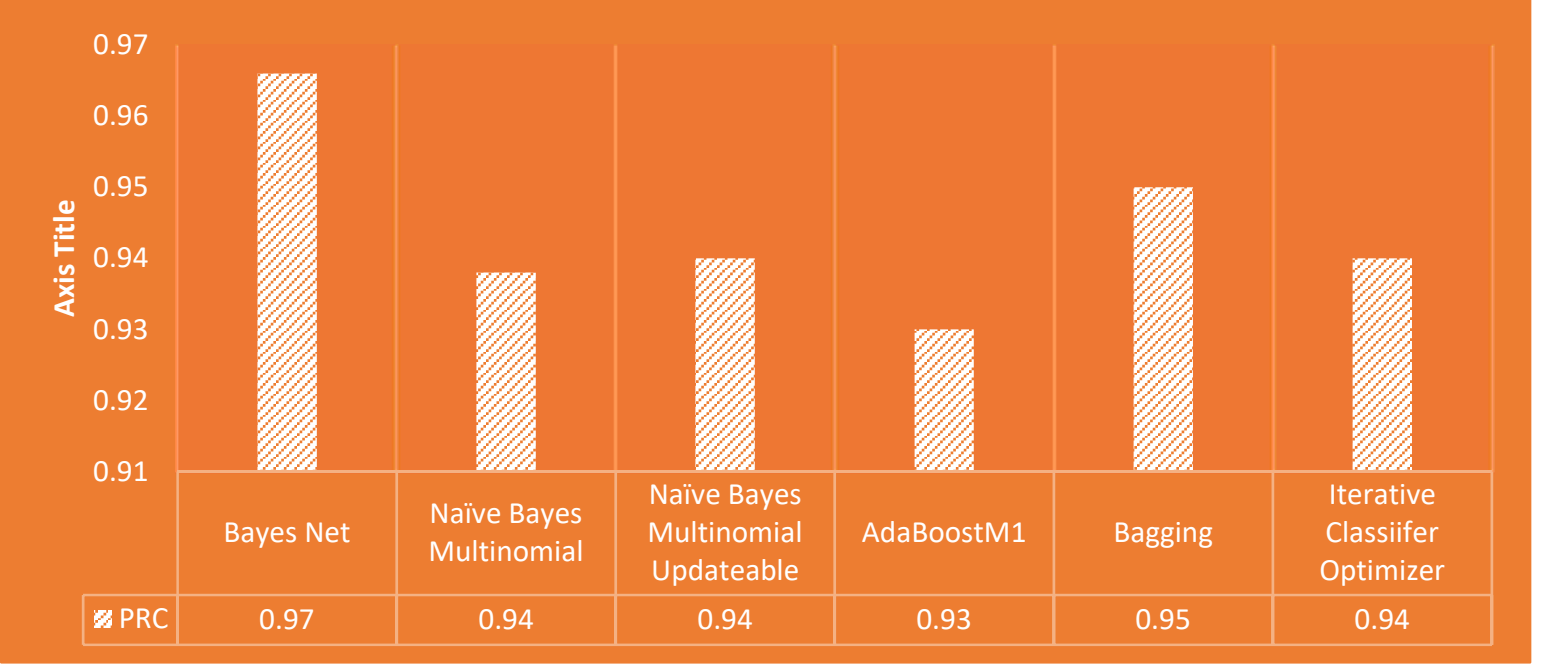

Figure 8: Performance of various classifiers with their PRC values

The above figure 8 shows that the Naïve Bayes Multinomial classifier and Naïve Bayes Multinomial Updateable classifier of Bayes category and AdaBoostM1 classifier of Meta category has same PRC values as well least PRC level which is 0.94 of PRC values. The Bayes Net is having 0.97 of ROC value which is highest PRC value. The Bagging classifier is 0.95 of PRC level. The Iterative Classifier Optimization is having 0.94 of PRC value.

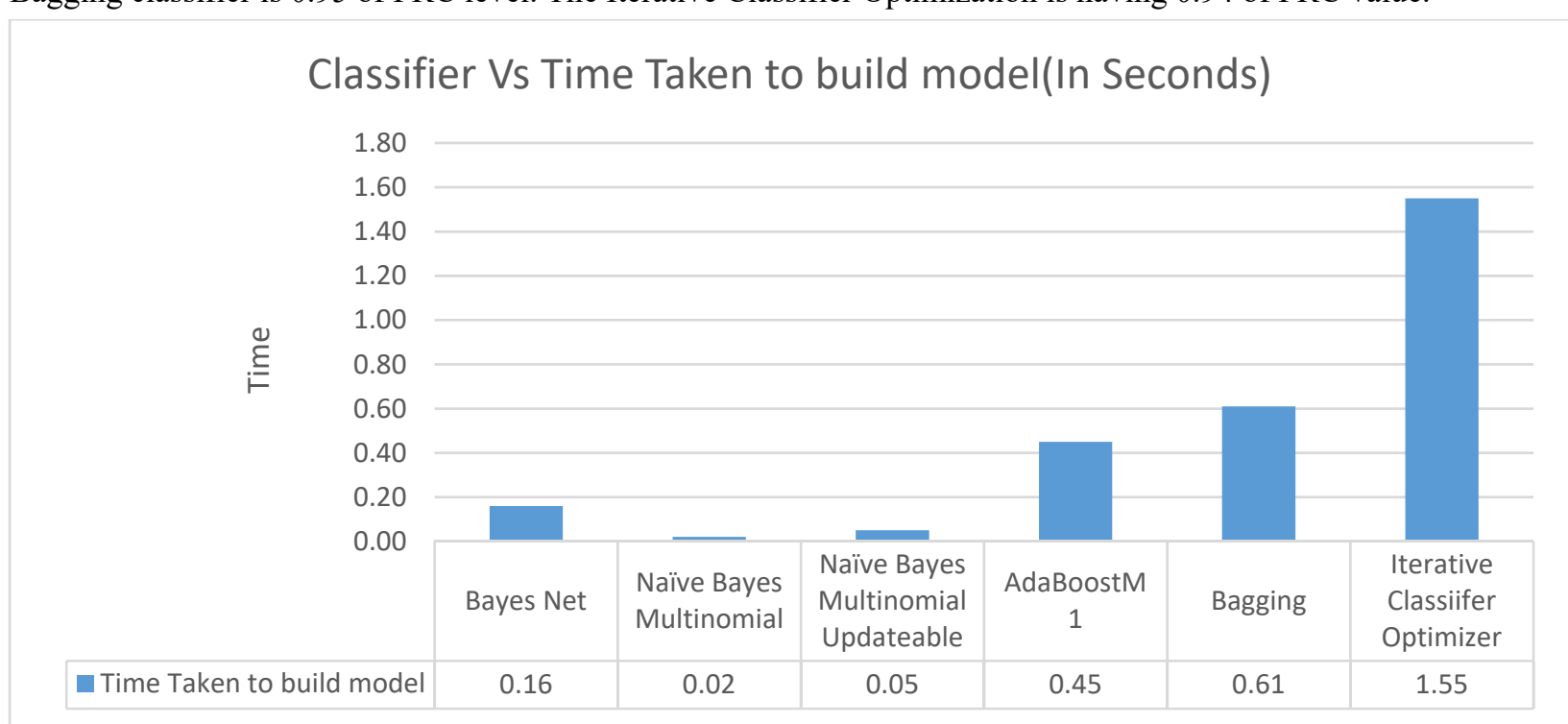

Figure 9: Performance of various classifiers with their time consumption to build models

The above figure 9 shows that the Naiive Bayes Multinomial classifier of Bayes category takes least time consumption to build a model which is 0.02 seconds, The Iterative Classifier Optimization is taking more time consumption to build a model which is 1.55 seconds.

\section{Conclusions}

The Iterative Classifier Optimization is having highest accuracy level which is $93 \%$ of accuracy. The highest kappa statistic value is 0.86 which is produced by Iterative Classifier Optimizer classifier. The least MAE value is 0.10 which is produced by Bayes Net classifier. The least RMSE value is 0.28 which is produced by Bayes Net classifier. The least RAE value is $19.73 \%$ which is produced by Bayes Net classifier and the least RRSE value is $56.59 \%$ which is produced by Bayes Net classifier. The Iterative Classifier Optimization is having highest precision level which is 0.93 of precision value. The Iterative Classifier Optimization is having highest recall level which is 0.93 of recall value. The Naïve Bayes Multinomial classifier of Bayes category takes least time consumption to build a model which is 0.02 seconds, The Iterative Classifier Optimization is taking more time consumption to build a model which is 1.55 seconds. This research works recommends that image feature extraction technique such as Auto color correlogram Filter techniques on Alzheimer's images dataset by implementing Itetrative Classifier Optimizer classifier of ensemble model. 


\section{References}

[1] Oh, K., Chung, YC., Kim, K.W. et al. Classification and Visualization of Alzheimer's Disease using Volumetric Convolutional Neural Network and Transfer Learning. Sci Rep 9, 18150 (2019). https://doi.org/10.1038/s41598-019-54548-6

[2] Grundman, M., Petersen, R. C. \& Ferris, S. H. Mild cognitive impairment can be distinguished from alzheimer disease and normal aging for clinical trials. Archives of Neurology. 61, 59-66 (2004).

[3] Davatzikos, C., Fan, Y., Wu, D. X. \& Shen, S. M. Detection of prodromal Alzheimer's disease via pattern classifcation of magnetic resonance imaging. Neurobiology of Aging. 29, 514-523 (2008).

[4] Suk., H. I., Lee, S. W. \& Dinggang, L. Deep ensemble learning of sparse regression models for brain disease diagnosis. Medical Image Analysis 37, 101-113 (2017).

[5] Hayit, G., Bram van, G. \& Ronald, M. S. Guest Editorial Deep Learning in Medical Imaging: Overview and Future Promise of an Exciting New Technique. IEEE Transactions on Medical Imaging. 35, 1153-1159 (2016).

[6] Litjens, G. A. Survey on Deep Learning in Medical Image Analysis. IEEE Transactions on Medical Imaging. 42, 60-88 (2017).

[7] Rashid, B. et al. Classifcation of schizophrenia and bipolar patients using static and dynamic resting-state fMRI brain connectivity. Neuroimage. 134, 645-657 (2016).

[8] Arbabshirani, M. R., Castro, E. \& Calhoun, V. D. Accurate classifcation of schizophrenia patients based on novel resting-state fMRI features. In: EMBC, https://doi.org/10.13140/2.1.1332.6723, 2014 (2014).

[9] Pinaya, W. H. L. et al. Using deep belief network modelling to characterize diferences in brain morphometry in schizophrenia. Scientifc Reports. 6, 38897 (2016).

[10] LeCun, Y., Bengio, Y. \& Hinton, G. Deep learning. Nature. 521, 436-444 (2015)

[11] Plis, S. M. et al. Deep learning for neuroimaging: a validation study. Frontier Neuroscience. 8, 1-11 (2014).

[12] Shen, D., Wu, G. \& Suk, H. Deep Learning in Medical Image Analysis. Annual Review of Biomedical Engineering. 19, 221-248 (2017).

[13] Payan, A. \& Montana, G. Predicting Alzheimer's disease: a neuroimaging study with 3D convolutional neural networks. In:ICPRAM. $355-362(2015)$.

[14] Vieira, S., Pinaya, W. H. L. \& Mechelli, A. Using deep learning to investigate the neuroimaging correlates of psychiatric and neurological disorders: method and applications. Neurosci Biobehav Review. 74, 58-75 (2017).

[15] Kim, J., Calhoun, V. D., Shim, E. \& Lee, J. H. Deep neural network with weight sparsity control and pre-training extracts hierarchical features and enhances classifcation performance: evidence from whole-brain resting-state functional connectivity patterns of schizophrenia. Neuroimage. 124, 127-146 (2016).

[16] Lee, G. et al. Predicting Alzheimer's disease progression using multi-modal deep learning approach. Sci Rep. 9(1), 1952 (2019).

[17] Liu, M., Zhang, J., Lian, C. \& Shen, D. Weakly Supervised Deep Learning for Brain Disease Prognosis Using MRI and Incomplete Clinical Scores. IEEE Transactions on Cybernetics, https://doi.org/10.1109/TCYB.2019.2904186 (2019).

[18] Krizhevsky, A., Sutskever, I. \& Hinton, G. Imagenet classifcation with deep convolutional neural networks. Neural Information Processing Systems. 25, 1097-1105 (2012).

[19] Bar, Y., Diamant, I., Wolf, L. \& Greenspan, H. Deep learning with non-medical training used for chest pathology identifcation. In: SPIE Medical Imaging, https://doi.org/10.1117/12.2083124 (2015).

[20] Abrol, A et al. Deep Residual Learning for Neuroimaging: An application to Predict Progression to Alzheimer's Disease. bioRxiv,https://doi.org/10.1101/470252 (2018).

[21] Fedorov, A. et al. Prediction of Progression to Alzheimer's disease with Deep InfoMax. arXiv:1904.10931 (2019).22. Rieke, J., Fabian, E., Weygandt, M., Haynes, J. D. \& Ritter, K. Visualizing Convolutional Networks for MRI-Based Diagnosis of Alzheimer's Disease. MICCAI. 24-31 (2018).

[22] Yang, C., Rangarajan, A. \& Rnaka, S. Visual Explanations From Deep 3D Convolutional Neural Networks for Alzheimer's Disease Classifcation. In: AMIA (2018).

[23] Korolev, S., Safiullin, A., Belyaev, M. \& Dodonova, Y. Residual and plain convolutional neural networks for 3D brain MRI classifcation. In ISBI, https://doi.org/10.1109/ISBI.2017.7950647 (2017).

[24] Hosseini-Asl, E., Keynton, R. \& El-Baz, A. Alzheimer's disease diagnostics by a 3D deeply supervised adaptable convolutional network. Frontiers In Bioscience. Landmark 23, 584-596 (2018).

[25] Kilian, H. et al. Multimodal Hippocampal Subfeld Grading For Alzheimer's Disease Classifcation, https://doi.org/10.1101/293126 (2018).

[26] Suk, H. I. \& Shen, D. Deep Learning-Based Feature Representation for AD/MCI Classifcation. In: MICCAI. 583-590 (2013).

\section{Author Profile}

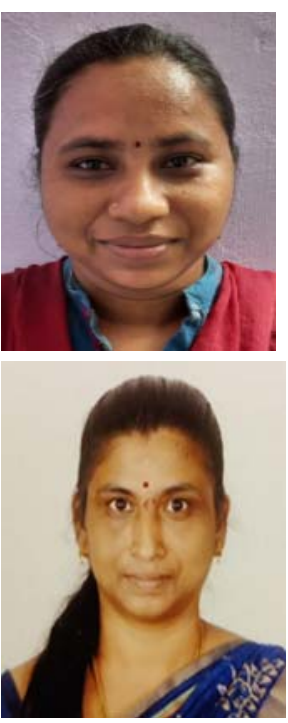

Ms. Deepa.D, received B.E degree in 2006 from Anna University, obtained M.Tech degree from BIHER in 2009 and doing research in Computer Science and Engineering in Bharath Institute of Higher Education and Research, Chennai, India. My current research interests are in Data Mining, Image Processing, Machine Learning, Artificial Intelligence, Data science.

Dr.ANITHA KARTHI received her B.E degree from the University of Madras, Chennai, India in 1998. She obtained her M.E. degree (First Class with Distinction) from CEG, Anna University, Chennai, India in 2007 and her Ph.D. degree from CEG, Anna University, Chennai, India in 2013. She is currently working as a Professor in the Department of Computer Science and Engineering, Bharath Institute of Higher Education and Research, Chennai, India. Her current research interests are in data science, machine learning, and IoT applications in the field of Electrical, Electronics, and Computer Science Engineering. 